\title{
LA POLÍTICA CUBANA FRENTE A LA COID 19. LA EDUCACIÓN EN ESTE CONTEXTO
}

\section{THE CUBAN POLITICS IN FRONT OF THE COVID 19. THE EDUCATION IN THIS CONTEXT.}

\author{
Nancy Lucía Chacón Arteaga ${ }^{1}$ \\ Universidad de Ciencias Pedagógicas \\ "Enrique José Varona \\ La Habana, Cuba
}

1 nchaconarteaga@gmail.com Orcid: https://orcid. org/0000-0003-2228-5505

Acumula más de 40 años de experiência docente. Es Licenciada en Ciencias Políticas en la Universidad de la Habana 1976. Doctora en C. Filosóficas en 1996 y Doctora em Ciencias 2011. Premio Nacional de Ciencias Sociales de la Academia de Ciencias de Cuba 2016. Titular Académica de C. Sociales de la ACC 2018. Profesora Emérito de la Universidad de C. Pedagógicas "Enrique José Varona" 1918, donde es Presidenta fundadora de la Cátedra de Ética Aplicada a la Educación desde 1997. Es Presidenta de la sub comisión nacional de planes y programas de la disciplina Educación para la vida ciudadana del Ministerio de Educación de Cuba. Es Profesora Honoraria de la Universidad Autónoma de Santo Domingo UASD 2010. Dirije proyectos de investigaciones sobre Ética aplicada a la Educación moral, em calores y ciudadana. Es membro del Comité Nacional Cubano de Bioética. Há publicado vários líbros y artículos em revistas científicas. Por su trayectoria professional há merecido numerosos prêmios y condecoraciones, entre ellas: Distinción por la Educación cubana, Premio a la mejor tesis de 2do grado científico defendida em 2011 por el Ministerio de Educación Superior de Cuba MÊS, Premio Exaltaxión al Mérito Pedagógico por la Red Iberoamericana de Pedagogía REDIPE 2016, Premio Eureka 2016 del Consejo mundial de académicos universitários COMAU. Distinción de la Estralla Martiana 2017 por el Sindicato Nacional de Trabajadores de la Educación, la Ciencia y el Deporte SNTECD.

\section{RESUMEN}

El trabajo deriva del proyecto de investigación sobre la "Educación para la vida ciudadana en la formación docente", en el análisis de la política cubana de enfrentamiento de la Covid 19 y el lugar y papel de la Educación en este contexto.

\section{PALABRAS CLAVE:}

Enfrentamiento a la Covid19, Educación, valores

\section{ABSTRACT}

The work presents a result of the project of investigation on the "Educación for the citizen life in the docente" formation, in the analysis of the Cuban politics of |enfrentamientol of the Covid 19 and the place and paper of the education in this context. 


\section{KEY WORDS:}

Enfrentamiento to the Covid19, education, values

\section{INTRODUCCIÓN NECESARIA}

José Martí (1853 - 1895), el apóstol de la independencia de Cuba desarrolló en su corta y fecunda vida un pensamiento revolucionario - humanista, universal y visionario, que desde una concepción política, ético - moral, jurídica, estético - artística, científica, filosófica y religiosa, que le permitió entre sus urgencias entregar todo su empeño en aras de organizar y llevar a cabo la guerra necesaria de independencia contra el colonialismo español, pero a su vez proyectar la necesidad de la Educación de las nuevas generaciones de cubanas y de Nuestra América que expresó en su ideario pedagógico:

"Al mundo nuevo corresponde la Universidad nueva. A nuevas ciencias que todo lo invaden, reforman y minan nuevas cátedras. Es criminal el divorcio entre la educación que se recibe en una época, y la época.

Educar es depositar en cada hombre toda la obra humana que le ha antecedido: es hacer a cada hombre resumen del mundo viviente, hasta el día en que vive: es ponerlo a nivel de su tiempo, para que flote sobre él, y no dejarlo debajo de su tiempo, con lo que no podrá salir a flote; es prepararlo para la vida" (Martí, J.1975)

Esta concepción de Educación de José Martí en la obra educacional de la Revolución cubana ha sido una realidad a partir del 1 ro de enero de 1959, al tener en cuenta tal y como se reconoce por el Líder histórico, el comandante invicto Fidel Castro Ruz, desde su histórico alegato de la "Historia me absolverá"(1953), que la educación es un derecho inalienable del ser humano, del pueblo, lo cual es respaldado por la voluntad política desarrollada por el Partico Comunista de Cuba y Gobierno revolucionario, plasmado en la Carta Magna de la sociedad cubana, que en su sentido martiano recoge el pensamiento cívico de Martí cuando dice: "Yo quiero que la ley primera de la República sea el culto de los cubanos a la dignidad plena del hombre"(Martí, J. 2019)

El trabajo tiene como objetivo valorar la importancia de la educación para la vida ciudadana en el contexto educativo cubano, su lugar y papel en el enfrentamiento de la Covid 19 y post Covid.

\section{LO QUE DEJÓ AL DESCUBIERTO LA COVID 19}

La humanidad vive hoy la tragedia de una pandemia de alto riesgo letal, el sar - cov 2 o nuevo coronavirus covid 19 , que ha puesto a prueba todos los resortes y mecanismos con que cuentan los seres humanos en el planeta para hacer frente a la misma, según la organización y gestión que para ello han asumido los diferentes sistemas socio políticos - económicos de los gobiernos y estados de los países a escala global.

Sin embargo, los seres humanos hemos llegado a la segunda década del tercer milenio con una deuda humana y planetaria que arrastra desde el Siglo XX y que ninguna de las metas "humanistas", "medioambientalistas" o "educativas" para el desarrollo sostenible de la UNESCO o de la UNICEF, entre otros organismos internacionales para proyectar metas de desarrollo para la humanidad, como lo hace ahora la agenda 2030 , se han logrado vencer de forma homogénea o al unísono en todos los países por igual.

Muchos son los factores y causas que en forma de barreras se levantan para impedir estos propósitos del desarrollo sostenible de los seres humanos y el planeta. Las más profundas están en el desarrollo desigual de las naciones engendrado por el sistema capitalista, que en sus diferentes fases de 
desarrollo llega hoy al capitalismo salvaje de ideología neoliberal, colonizadora, guerrerista, xenofóbica y hegemónica de las potencias imperialistas, encabezadas por los Estados Unidos, el que ante el desarrollo científico y tecnológico que ha permitido la globalización por medio de la digitalización de las sociedades, la comunicación y trasferencias de tecnologías, entre otros procesos, también aspira a un supra gobierno global, con el dominio de las riquezas naturales del planeta y una "ciudadanía global o planetaria" sin patria, identidades, ni fronteras que respondan a sus intereses egoistas.

Obviamente estos propósitos apuntan al despliegue de una nueva estrategia geopolítica para la redistribución de áreas de influencias por las riquezas de los países, que borre fronteras, subsuma o absorba identidades nacionales $y$ culturales, como pasa ya hoy con las poblaciones autóctonas, las mal llamadas "minorías étnicas", la tragedia humana de los emigrantes de todo el planeta, así como la satanización de todos los movimientos, asociaciones y organizaciones sociales que se rebelen ante el orden mundial establecido y sus reglas de doble rasero jurídico - moral, siempre a favor de los poderosos, para lo cual se emplean todos los medios tecnológicos y mediáticos para construir realidades virtuales, falsos positivos, escenarios bélicos, así como el empleo de las ciencias, las ingeniería biológica, la biotecnología, etc, en experimentos con poblaciones humanas y en las guerras, sembrando el poder bajo el signo del terror, la muerte, el castigo, el desprecio por el ser humano, la vida y el planeta tierra.

Las políticas neoliberales aplicadas, en particular, las de la región de la América Latina y el Caribe, durante décadas y a través de gobiernos de derecha que se han dejado llevar por los cantos de sirena del "sueño americano" y los de sus propios intereses egoístas, han desmembrado el poder del estado para centrarse en el mercado, el consumo, la privatización y las pautas dictadas a los países endeudados y dependientes por el FMI, BFI, lo que ha llevado al desmontaje de los sistemas de salud, el recorte de los seguros y la seguridad social, la privatización de la Educación, entre otros despojos, dejando este vital asunto a la suerte de cada quien en una especie de "sálvese quien pueda", donde los que tienen recursos y solvencia, empezando por las clases adineradas y las élites del poder, estarán en mejor posición para salvarse ante una situación tan drástica como la de la pandemia por la que estamos atravesando y ha costado millones de contagiados y fallecidos.

Lamentablemente es predominante esta situación en la región, incluso están vivos los ejemplos de varios países que después de haber pasado por las vivencias de gobiernos en los que la educación y la salud fueron atendidos con una concepción de universalidad en interés de los derechos del pueblo, como en los casos de Brasil (I. Lula), Ecuador (E. Correa), Argentina (C. Fernández) Bolivia (E. Morales), en los que se experimentaron ciertos avances en estos terrenos, ahora están sumidos en una catástrofe sanitaria ante el colapso de las insuficientes estructuras de salud, la no cobertura de seguros, la incompetencia gubernamental demostrada para el manejo de la pandemia y las no garantías de salud de sus pueblos, que son entre otros, los que más han aportado a las cifras descomunales de contagios y fallecidos en cementerios improvisados, incluyendo la afectación del personal de la salud sin protección institucional.

Puede decirse que en este escenario se revelan tanto los estragos de la pandemia de la Covid19 por su alta contagiosidad y letalidad, así como los impactos negativos de los gobiernos neoliberales, su incompetencia política y la falta de sustentabilidad de sus sistemas sociales (de salud, educación y seguridad social), de desprecio al ser humano y el medio ambiente, 
xenofóbico y antisolidario, los que constituyen una fragrante violación de los derechos humanos, cuyas posiciones de algunos presidentes fue la de minimizar la pandemia, no propender a la protección de sus ciudadanos, ni al cumplimiento de medidas sanitarias, con el fin de salvar a toda costa la economía de las clases altas y el empresariado, sobre las muertes de la población pobre, de los ancianos que consideran una carga social, de las poblaciones indígenas en sus asentamientos, de los emigrantes, entre otros sectores desposeídos, de frente al colapso de la salud pública y ninguna garantía o seguridad social.

No obstante, durante estos tiempos de confinamiento y aislamiento social, ante todo ello se ha alzado la voz del género humano que recorre latitudes contraponiéndose a todo odio, a crímenes racistas como el del afroamericano Jeorge Floyd en Minnesota, EEUU, cuyo estallido de protestas desatadas expresa la esencia supremacista y arrogante que históricamente ha caracterizado no solo la política interracial en Norteamérica sino en todo el mundo.

Aún en medio de la crisis de la pandemia, no cesa la práctica del terrorismo de estado y el paramilitarismo en el asesinato selectivo de los líderes sociales en la región como el caso de Colombia en la etapa de "post conflicto" después de firmado el acuerdo de Paz entre el gobierno de Juan Manuel Santo y las FARC en la Habana; la persecución judicial de los líderes de izquierda para sacarlos del escenario político, los golpes de estados e injerencia en los asuntos interna de los países como Venezuela, Nicaragua y Cuba, que son declarados por el gobierno de los EEUU y sus aliados, como el eje del mal; son solo algunos ejemplos de la situación geopolítica en la arena internacional.

Las voces cada vez más numerosas de los indignados se hacen sentir para cantarle a la vida, a la esperanza de un cambio a favor de la justicia social y equidad, a la necesaria solidaridad, cooperación y colaboración entre países para enfrentar la pandemia global, entre otros males, ante lo cual China, Rusia y Cuba, han seguido este camino y han actuado consecuentemente con el principio de la solidaridad

\section{EL EJEMPLO DE CUBA}

Cuba, un país bloqueado por más de seis décadas por la potencia más poderosa, agresiva y peligrosa del mundo, el imperialismo yanqui, a tan solo 90 millas de nuestras costas, ha resistido estoicamente todos los embates contra la Revolución cubana, cuyo sistema social socialista se sustenta en la defensa de los valores de la ideología martiana, marxista - leninista y fidelista, del patriotismo, la independencia y soberanía nacional, el antiimperialismo, la justicia social, la dignidad humana, la solidaridad y la unidad nacional, en la construcción ciudadana de una democracia con plena participación popular en todos los procesos y que desde su triunfo en enero de 1959, una de las tareas primeras junto con las económicas de la Reforma agraria, entre otras medidas populares, estuvo la epopeya de la Campaña de la alfabetización, declarándose Territorio libre de analfabetismo el 22 de diciembre de 1961, después de vencer en 72 horas (16 - 19 de abril 1961), la agresión mercenaria del gobierno norteamericano y la mafia cubano-americana, por Playa Girón, que significó la primera gran derrota del imperialismo yanqui en América. Cuba se crecía como un faro de luz que alumbraba las esperanzas de los desposeídos y pueblos oprimidos del mundo, haciendo realidad la idea martiana de que "Ser culto es el único modo de ser libre".

Unido a ello, como parte de una concepción revolucionaria integral de la plataforma programática de las transformaciones a llevar a cabo en la sociedad cubana, desde fecha temprana el Líder histórico de la Revolución Fidel Castro, en su visión estratégica del desarrollo del país, consciente de las condiciones heredadas tras cinco décadas de un capitalismo nacional 
dependiente y una deformación estructural de la economía monoproductora y exportadora del azúcar de caña, hacia el principal mercado los EEUU -quien cortó la compra de la cuota azucarera, rompió relaciones e impuso su genocida bloqueo hasta nuestros días- se proyectó por la necesidad de que en Cuba "el futuro de nuestra Patria tiene que ser necesariamente un futuro de hombres de ciencia"2(Castro, F. 1960).

Desde esa concepción se formaron los recursos humanos y el potencial científico cubano, creándose paso a paso las bases del desarrollo científico - tecnológico, fármaco biotecnológico y médico, con que contamos hoy y el sistema nacional de Educación general, politécnica y laboral, la Educación Técnica Profesional, la Educación artística, la Cultura física y el deporte, Escuelas formadoras de maestros primarios, la Educación especial y la Educación superior que abarcó gradualmente todas las ramas y áreas del saber de las ciencia, la tecnología, las humanidades, la cultura física y las artes, entre ellas las Ciencias agrónomas, las Ciencias técnicas - ingenieriles, de las comunicaciones y la información, las Ciencias médicas, entre otras, así como la formación de los Licenciados en Educación para todos los niveles de enseñanza del país. La educación, la salud y el deporte, son derechos inalienables del pueblo con acceso público y gratuito.

\section{EL ENFRENTAMIENTO A LA COVID 19}

El desarrollo alcanzado por la sociedad socialista cubana tiene una expresión jurídica expresada en la nueva Constitución (aprobada posterior a la consulta popular el 24/2/2019 por la Asamblea Nacional del Poder Popular), en la que se profundiza y amplían los alcances de la participación democrática, los deberes y derechos ciudadanos, la justicia social, así como refrenda los cambios del modelo socio-económico, la nueva organización y funcionamiento del sistema político de gobierno y del estado cubano.

Hoy, son las nuevas generaciones formadas en y por la Revolución, quienes han asumido la alta responsabilidad de la dirección del país junto al PCC y el pueblo, representando el núcleo de la unidad nacional que nos distingue, lo que significa la continuidad de la generación histórica y del Líder Fidel Castro, para llevar adelante el socialismo y su permanente perfeccionamiento, en la lucha por una sociedad prospera, sostenible y por un mundo mejor.

El anuncio de la amenaza del nuevo coronavirus sar-cov2 que provoca la Covid 19, surgido en la ciudad China de Wahun en diciembre del 2019, fue la señal para que en Cuba se monitoreara esta situación epidemiológica y las orientaciones de la OMS, hasta su declaración de pandemia global. Desde sus inicios ya en el país se daba un seguimiento noticioso y de información a la población de las previsiones y medidas tomadas por el gobierno y ministerio de salud pública para hacer frente a la pandemia cuando se detectara la entrada del primer caso a Cuba por la frontera, es decir se preparaban las condiciones y las reservas para esta batalla por la vida.

En la dirección de este proceso se creó el grupo de gobierno temporal de trabajo GTT, presidido por el Presidente de la República de Cuba, Miguel Díaz - Canel Bermudez y el Primer Ministro Manuel Marrero, para el enfrentamiento a la pandemia con la participación del consejo de ministros y las fuerzas y sociales que le dieron un carácter intersectorial y multidisciplinario, articulándose de forma armónica la política, la ciencia y la tecnología, en la elaboración de una estrategia certera donde la información, la comunicación e interacción diaria con el pueblo por los medios de comunicación en particular la TV, así como el accionar de los consejos de defensa provinciales y municipales, 
desempeñaron un importante papel en la conducción de esta batalla para salvar vidas.

En el artículo publicado por el Presidente Dr. C. Miguel Díaz -Canel Bermudez en coautoría con el Dr. C. Jorge Núñez Jover, Académico Titular de C. Sociales de la Academia de Ciencias de Cuba, se condensa una valiosa información acerca de la "Gestión gubernamental y la ciencia cubana en el enfrentamiento a la COVID-19" en el que se describe entre otros pasos, medidas y acciones desarrolladas las siguientes:

- "Reunión semanal con expertos y científicos para evaluar los resultados de las investigaciones y su aplicación.

- Activación de los Consejos de Defensa Provinciales en todo el territorio nacional.

- Conferencias de prensa diarias para informar al pueblo sobre la actualización de la marcha del Plan para la

Prevención y Control del nuevo coronavirus (COVID-19).

- Mesas Redondas diarias para

actualizar sobre diferentes

temas relacionados con el Plan para la

Prevención y

Control de la COVID-19.

- Sesión de trabajo semanal con el

Grupo Asesor

Económico para abordar los temas concernientes a la estrategia de desarrollo económico social. "(Díaz-Canel, M. 2020)

La estrategia epidemiológica - sanitaria, contó con tres etapas, cada una con medidas sanitarias y protocolos médicos de procedimientos para la detección y atención de los casos positivos que eran ingresados en los hospitales o salas de hospitales habilitados para su atención, así como la detección de los anillos de contactos de contactos y de contactos, los cuales iban a centros de aislamientos para hacerles las pruebas y estar el tiempo reglamentario hasta confirmarse que no eran positivos para regresar a sus domicilios.

Muchos voluntarios de centros de trabajos, docentes y estudiantes universitarios, y organizaciones de masas de las comunidades, entre otros, se sumaron a colaborar en estos centros de asilamiento e incluso en algunos hospitales, previa preparación de las medidas de bioprotección y protocolos a seguir en el rol que cada uno desempeñaría como personal de apoyo, así como pasar la prueba del PCR en tiempo real para confirmar su estado de salud a la entrada y al cabo del período de labor a su salida pasaban 15 días de aislamiento, descanso y nuevamente la prueba del PCR para ir hacia sus casas e integrarse sin riesgo a su seno familiar.

En este riguroso y sistemático trabajo, Cuba aporta un modelo con una metodología que desarrolla en un procedimiento novedoso que son las pesquisas diarias para encontrar entre la población que no entraban en los círculos de los contactos, las posibles personas infectadas, este proceso se desarrolló por el sistema de atención primaria del médico y enfermera de la familia en todos los territorios y localidades, incorporándose los estudiantes y docentes de las escuelas de medicina, los médicos estomatólogos entre otro personal a fin, que cada día tocan puerta a puerta de la vecindad. Esta acción es respaldada por la creación cubana de una aplicación para el móvil que facilita a quienes desean realizar su autopesquisaje de salud y registrar sus síntomas, los que también son atendidos medicamente.

A pesar del genocida bloqueo y persecución por parte del gobierno de los EEUU que impedía que Cuba adquiriera los materiales médicos necesarios para enfrentar esta pandemia ya sean por donaciones o comprados, en el país se contaba con la reserva, los medicamentos algunos de ellos de producción cubana, 
respiradores, entre otros materiales necesarios para la atención a toda la población que lo necesitara, incluyendo extranjeros residentes o turistas en el país, totalmente gratuito como son los servicios médicos en Cuba, en tal sentido el sistema de salud cubano da una cobertura total sin colapsar por falta de materiales, médicos y paramédicos, ni instalaciones hospitalarias o centros de asislamientos.

\section{EL PRINCIPIO DE LA SOLIDARIDAD Y EL INTERNACIONALISMO}

Unido a todo ello Cuba se dispuso a llevar su solidaridad y cooperación médica a los países necesitados que solicitaran ayuda, para lo cual se cuenta con los médicos y para médicos del Contingente Internacional de Médicos Especializados en Situaciones de Desastres y Graves Epidemias "Henry Reeve”, creado por Fidel en agosto del 2005 (en ocasión del huracán Katrina, que afectó el sur de los EEUU los que rechazaron el ofrecimiento de ayuda cubana), que son la máxima expresión del histórico principio de la práctica solidaria e internacionalista de la política revolucionaria, que tiene como antecedentes desde tan temprana fecha de mayo de 1960, en ocasión del terremoto que devastó al pueblo chileno, el envío de una brigada de médicos, acompañada con una carga significativa de materiales médicos, insumos, alimentos y ropas para los damnificados; tal como definió Fidel en la ética humanista y valores revolucionarios, para Cuba la solidaridad no es dar lo que le sobra, sino compartir lo que se tiene.

La Brigada Henry Reeve acumula una larga trayectoria de misiones en condiciones de riesgos de desastres y epidemias llevando el amor solidario y el aliento de salvar vidas, en la lucha contra la Covid19 tiene presencia en más de 35 países a las más de 45 solicitudes realizadas. La alta valoración del ejemplo de la solidaridad humana que en estos tiempos tan difíciles representa el valory altruismo de la Henry
Reeve, ha levantado el interés internacional de varias organizaciones y personalidades, de hacer un movimiento a favor de la propuesta para que le sea otorgado el Premio Nobel de la Paz.

\section{LA EDUCACIÓN EN TIEMPOS DE COVID 19}

Sin educación no hay Revolución, esta máxima del pensamiento estratégico y humanista de Fidel, está en la transversalidad de este artículo, ya que sin una preparación sólida sustentada en el sistema educativo cubano no pudiera hablarse de un enfrentamiento estratégico e integral a la pandemia en el país, ya que todos los recursos materiales, científicos y tecnológicos puestos en función en una unidad de pensamiento y acción como país, son resultados de una obra educacional en la formación y preparación de los recursos humanos competentes y con los valores que se requieren para los modos de actuación profesionales y como ciudadanos de toda la población.

Quiere decir que la educación está en la base de la cultura multidimensional creada por la Revolución y una conciencia patriótica, antimperialista, responsable, solidaria e internacionalista, que posibilitó la articulación necesaria de todos los factores para el enfrentamiento y control de la pandemia en el país.

Pero la educación no solo está en esa base cultural, sino que acompaña todo el proceso de preparación de la población para este enfrentamiento no solo por las medidas tomadas, sino por la necesaria comprensión y conocimiento de causa, la Educación como vía para la prevención y el enfrentamiento, no solo en los mensajes de bien público, el sistema de información interactivo del desarrollo de la estrategia, la marcha y control de la pandemia por múltiples vías, son también acciones de un valor altamente educativo. 
El sistema de educación aún con un grupo de medidas después de declarada la entrada de la Covid19 al país, mantuvo las escuelas abiertas hasta finales del mes de marzo (24), en que se decide cerrar las escuelas, aunque mantener con un horario flexible (9am-3pm) los círculos infantiles para dar servicio a las madres trabajadoras que estaban en puestos imprescindibles de trabajo y no tenían con quien dejar a sus hijos en casa. Cabe señalar que el estado cubano garantizó el salario de los trabajadores en condiciones de asislamiento social, por los conceptos de tele trabajo, trabajo a distancia o semi presencial, en el caso de los docentes de todos los niveles de enseñanza recibimos el salario devengado.

En ese momento la escuela cubana ya había abarcado aproximadamente en todos los niveles educativos un $70 \%$ del contenido del curso, lo que posibilitó que se pudiera dar una continuidad de clases televisivas y actividades educativas orientadoras de cada asignaturas por grados en una programación que alternaba en los días de la semana, las sesiones matutinas y vespertinas en tres canales de la TV nacional (Canal educativo, Canal educativo 2 y Canal Tele rebelde), a disposición para las mismas, organizado por el Ministerio de Educación, con la participación de los metodólogos nacionales, los tele profesores, entre otras entidades como la empresa de Cinesoft, que apoyó con materiales audiovisuales.

Esta rápida respuesta del MINED posibilitó que los educandos dieran una continuidad a sus estudios, mantener sus motivaciones y hábitos educativos, así como fortaleció el papel de la familia que apoyó este seguimiento del estudio y tareas de sus hijos en las casas; esta actividad educativa no comprometió los nuevos contenidos, las evaluaciones, ni el pase de grado de los estudiantes, lo que siempre se previó para el momento en que se decidiera en la estrategia de país, la reanudación del curso para retomar las clases con los contenidos y objetivos pendientes y el cierre evaluativo del curso $19-20$ y el inicio del nuevo curso $20-21$.

Esta estrategia concebida por el sistema educativo cubano, además de ser una rápida respuesta en la organización, tuvo una amplia difusión e información que dio seguridad a los educandos, a las familias, a los maestros y a la sociedad toda, con una base orientadora de los por menores no solo se cómo se desarrollaría esta etapa de estudios durante la pandemia, sino de cómo en la reanudación, se tratarían los contenidos que faltaban de las asignaturas y se realizarían las evaluaciones correspondientes para el cierre del curso.

En los grados terminales se realizarían las graduaciones correspondientes, en el caso de la continuidad de estudio del 9no grado, se llenarian las boletas de solicitudes para hacer el escalafón de las provincias y realizar el otorgamiento de las especialidades de estudios. En el caso del 12 grado, que tuvo la continuidad de su preparación para los exámenes de ingreso a la universidad por la misma vía televisiva, realizarían sus exámenes para clasificar en el escalafón provincial y proceder al otorgamiento de las carreras.

De esta forma se concibe que cuando se entre en la etapa de reinicio de las clases, se conciben 10 semanas para continuidad y cierre del curso 19 - 20 y posterior a ello el inicio del curso 20 21 que se realizaría en menos semanas que el fondo de tiempo habitual, en este caso es de 38 semanas.

Esto conllevó a la convocatoria por parte del MINED de los directores de los niveles educativos (Educación primaria, Secundaria Básica, Pre universitario, Educación de Adultos, la Educación Técnica y Profesional y las Escuelas pedagógicas), a los metodólogos nacionales de las disciplinas, a los presidentes de las comisiones nacionales de los planes 
y programas de estudios de las diferentes disciplinas y a los docentes de los colectivos de autores de los programas y libros de textos escolares de las mismas, acompañados por los directivos del Instituto Central de Ciencias Pedagógicas ICCP y los Presidentes de las comisiones de la Universidad de Ciencias Pedagógicas "Enrique José Varona", para que a partir de la remodelación que se había hecho con vista al perfeccionamiento del sistema educativo, que debía empezar a generalizarse en el curso 20 - 21 (proceso que quedó aplazado hasta que existan las condiciones para implementar la transformación curricular elaborada y experimentada en escuelas del país), se hicieran los reajustes a los programas vigentes con las actualizaciones y/o modificaciones posibles, para introducirse en el desarrollo del curso en el tiempo previsto y atendiendo a las exigencias de las medidas y protocolos de las condiciones de postpandemia en que se desarrollará dicho curso escolar.

Los reajustes implican que los programas tienen una cierta reducción en horas clases, las que se realizarán en días y sesiones alternas para cada grado, por lo que los estudiantes no irán todos los días en doble sesión a la escuela, sino a las que les corresponda a su grado, según la planificación y organización escolar, para cumplir con el distanciamiento que requieren los procesos educativos en la escuela en estas excepcionales circunstancias, para ello se mantiene el sistema de apoyo de actividades educativas y clases por la TV según se diseñe por las frecuencias de horas clases que tiene de cada disciplina en el reajuste de los programas y su dosificación en el curso; a la vez las Direcciones de educación provinciales y los gobiernos de cada territorio, realizan un gran esfuerzo en la remodelación constructiva de algunos centros educativos para alistar las condiciones y cumplir estos los requerimientos establecidos en el reinicio de las clases.
Obviamente que la puesta en práctica de estos reajustes curriculares, solo serán posibles con el acompañamiento previo de la preparación metodológica y didáctica de todos los directivos y el personal docente que deben llevar a cabo el desarrollo de estos contenidos en sus escuelas, así como de las estructuras provinciales y municipales de Educación en todo el país, lo cual está previsto realizar como es habitual en la semana de incorporación de los docentes posterior a sus vacaciones y antes del inicio del curso escolar, aunque ya desde la primera etapa de la nueva normalidad en el mes de julio 2020 se inician las capacitaciones a nivel nacional por vía televisiva para precisar los reajustes curriculares que incluyen la evaluación y las nuevas formas de trabajos a los diferentes niveles educativos.

En el caso de la Educación superior, al tiempo del reinicio del curso se prevé 11 semanas para culminar las disciplinas que quedaron pendientes y sus evaluaciones del 2do semestre interrumpido por la pandemia y a continuación se da inicio al curso $20-21$, con unas 30 semanas, por lo que los jefes de disciplinas y docentes de cada universidad realizan los reajustes de sus programas para adaptarse a esta reducción de horas lectivas.

En este caso los docentes durante la etapa de pandemia y asislamiento social, han trabajado en los informes de la validación de los programas de todas las disciplinas de las carreras del nuevo plan de estudio $E$ que ya han culminado el 4to y último año de ejecución (tiempo de la cohorte primera edición de las carreras) y sobre la base de las experiencias y sugerencias de mejoras de los propios docentes, se han hecho las modificaciones y el perfeccionamiento de los programas que se desarrollarán aunque reajustados en horas en este nuevo curso escolar post pandemia 20-21. Para esta labor los colectivos de las comisiones nacionales de las carreras trabajaron a distancia por via de 
grupos de whatsap y el correo electrónico para el intercambio de ideas y documentos elaborados,

Puede decirse que en Cuba la política de gobierno en el enfrentamiento a la Covid 19, desarrolló una estrategia que dirigida por el Grupo gubernamental de trabajo Temporal GTT, logró articular el consejo de ministros, el sistema de defensa y de seguridad nacional con sus consejos de defensas provinciales, que garantizaron el aseguramiento, seguimiento, evaluación y control de todas las medidas paso a paso en cada etapa, proceso que se caracterizó por la transparencia e interacción con la ciudadanía, lo que contó con la unidad y apoyo del pueblo.

Cabe señalar que no siempre la ciudadanía manifestó la necesaria percepción del riesgo ante las características de alta contagiosidad, letalidad y asintomaticidad en un significativo $\%$ de personas diagnosticadas como positivas, por lo cual algunos de forma irresponsable incurrían en el incumplimiento de las medidas de aislamiento y protección epidemiológicas, no obstante, siempre tuvieron un tratamiento persuasivo, preventivo, informativo y educativo para corregir los comportamientos inadecuados, los que en ocasiones según la magnitud de la transgresión también se aplicaron multas o procesamiento judicial por incurrir en el delito de violaciones de la Constitución sobre riesgo y propagación de epidemias.

En general el sistema de salud cubana y el sistema educativo en una mancomunidad de las conquistas, garantías y seguridad social del pueblo cubano, demostraron su efectividad en la cobertura total que dan al pueblo sin distinción ni discriminaciones de ningún tipo, en un sistema socialista donde el ser humano tiene su justo valor en la dignidad humana, que ha logrado una vez salir más adelante pese los daños humanos, económicos y sociales provocadas por el impacto nefasto del genocida bloqueo yanqui, arreciado en tiempos de pandemia, con el ansiado fin de acabar con el pueblo cubano y su Revolución de conquistas sociales.

$\mathrm{Ni}$ el silenciamiento de los éxitos y logros de Cuba en su enfrentamiento a la pandemia por los grandes medios de la información global, las campañas de descréditos sobre las fortalezas educativas y científicas cubanas, así como de sus médicos solidarios, ante el hecho real de la Brigada Henry Reeve en más de 30 países del mundo salvando vidas, pueden esconder las verdades y razones del pueblo cubano demostrados en el modelo gubernamental sistémico e integral del enfrentamiento a la Covid19, con métodos de dirección democráticos y estilos de gestión y de participación ciudadana en una responsabilidad compartida y comprometida con el logro de los mejores resultados y la preparación de todos para transitar por las etapas de la recuperación gradual y asimétrica de la "nueva normalidad", como se denomina el restablecimiento de la dinámica y funcionamiento de toda la sociedad, entre medidas organizativas y protocolos sanitarios para cuidar no tener rebrotes de la pandemia.

En todo ello la Educación sigue ocupando un lugar estratégico como sustento del desarrollo cultural y humano de los cubanos y cubanas de estos tiempos y los venideros con preparación, entereza y amor, para la realización de sueños y esperanzas, hacer valer la justicia, la dignidad humana, la solidaridad y la paz en Cuba y el mundo.

\section{REFERENCIAS BIBLIOGRÁFICAS}

Martí, J. (1975): Obras completas en 28 tomos. Editorial C. Sociales. La Hanana. T.8, P.288

Constitución de la República de Cuba (2019): Edición Consejo de Estado de la República de Cuba. La Habana. PXVI.

Castro, F. (1960): Discurso pronunciado en el acto celebrado por la Sociedad Espeleológica de 
Cuba, en la Academia de Ciencias. La Habana. P. 3

Díaz - Canel, M. (2020): Gestión gubernamental y la ciencia cubana en el enfrentamiento a la COVID-19. Revista Anales de la Academia de Ciencias de Cuba. La Habana. P. 205

Castro, F. (2006): Fragmentos de los discursos publicados desde 1959 - 2006 en Ética y valores humanos. Multimedia CESOFTE, Universidad de C. Pedagógicas "Enrique J. Varona, la Habana.

Chacón, N. (2013): Martí y Fidel en el código de ética del educador cubano. Editorial Pueblo y Educación. La Habana.

Chacón, N. et.al. (2015): Educación en valores. Retos $\mathrm{Y}$ experiencias. Editorial Acuario. La Habana.

Chacón, N. (2017): Educación ética y en valores. Para una cultura de convivencia y paz, desde una perspectiva cubana. REDIPE. Colombia.

Chacón, N. et. al (2019): Aproximación a una concepción sobre la Educación para la vida ciudadana en la formación docente y en la escuela cubana. Editorial Félix Varela. La Habana.

Constitución de la República de Cuba (2019): Editora política. La Habana.

Chacón, N. (2020): Solidaridad global Vs. Hegemonismos en tiempos de la Covid19. Revista Iberoamericana de Docentes. España.

Díaz - Canel, M. y Núñez, J. (2020): Gestión gubernamental y la ciencia cubana en el enfrentamiento a la COVID-19. Revista Anales de la Academia de Ciencias de Cuba. La Habana.
Etapa de recuperación post Covid19 (2020): Medidas a implementar en sus tres fases. Tabloide informativo a la población. Editora política. La Habana.

Martí Pérez José. (1975) Obras Completas. Editorial C. Sociales, la Habana. 